\title{
Les médicaments tirés de la mer
}

Certaines particularités de l'écologie marine, telle la synthèse de substances de défense par des microorganismes marins, sont à la base de la découverte de molécules biologiquement actives dont un petit nombre est déjà utilisé en chimiothérapie anticancéreuse.

\section{Bernard Banaigs Louis Codomier Christian Francisco Jean Teste}

Groupe de recherches en biologie et chimie des végétaux marins, Université de Perpignan.

\section{RÉFÉRENCE}

1. Braekman JC, Daloze D. Les médicaments de la mer. La Recherche $1983 ; 143: 465-72$.

B. Banaigs, L. Codomier, C. Francisco, J. Teste : Université de Perpignan, avenue de Villeneuve, 66025 Perpignan cedex.

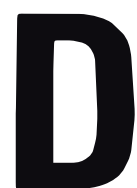
ès l'antiquité, la mer a fasciné l'humanité. Les aventuriers à la recherche de nouveaux territoires ont été, très tôt, à la fois attirés et terrifiés par cette immensité liquide et par les profondeurs océaniques aux insondables mystères. Pour mieux assurer sa subsistance, l'homme a appris de bonne heure à exploiter le domaine marin le plus accessible : pêche de poissons, mollusques, crustacés, etc. et récolte d'algues, notamment d'algues rouges des genres Gracilaria, Eucheuma, Chondrus... Très vite, les produits de cette pêche et de ces récoltes ont été utilisés pour d'autres buts que l'alimentation. C'est ainsi que bien avant notre ère, le " materia medica" chinois fait mention de préparations médicamenteuses à base de produits marins. Actuellement, la médecine populaire et la thalassothérapie n'utilisent que très peu de préparations médicinales à base de végétaux marins. Les recherches de molécules biologiquement actives n'ont débuté que vers les années 60 . Quelques pays (USA, Japon, Belgique, Australie et France) ont lancé des programmes de recherche sur le milieu marin. C'est le cas du National Sea Grant Program aux États-Unis qui associe firmes pharmaceutiques (Upjohn...), instituts nationaux (NCI et $\mathrm{NIH}$ ) et laboratoires universitaires (chimistes, biologistes, écologistes, pharmacologues, etc.). En France, deux programmes ont vu le jour : le projet Pharmocéan du Centre National pour l'Exploitation des Océans (CNEXO), et surtout le programme SNOM (Substances Naturelles d'Origine Marine) regroupant le CNRS, l'ORSTOM et la Société Rhône-Poulenc. Cet engouement pour la pharmacologie marine avait pour origine d'une part la découverte en 1969 d'un taux important $(1,5 \%$ du poids sec) d'une prostaglandine $\left(\mathrm{PGA}_{2}\right)$ chez une gorgone, et d'autre part la mise en évidence d'un fort pourcentage d'extraits d'organismes marins à activités antitumorales $(5$ à $8 \%$ contre $2 \%$ pour les végétaux terrestres) ou cardiovasculaires $(15 \%)$. Malgré les résultats fort encourageants concernant l'activité biologique de ces substances, la plupart de ces programmes de recherche furent arrêtés au début des années 8o. Après l'enthousiasme, ce fut le désenchantement! Un article de synthèse, paru dans La Recherche en avril 83 [1] a rendu compte de l'ensemble de ces travaux. Heureusement, le maintien du programme américain aboutira cependant à la découverte de nouvelles structures présentant des activités pharmacologiques significatives. A l'heure actuelle, un réel regain d'intérêt pour 
ces recherches marines existe en France et cet article s'efforce de rendre compte des résultats les plus récents dans ce domaine.

\section{Agents antiviraux et antinéop/asiques}

Parmi les très nombreuses molécules extraites d'organismes marins, et reconnues pharmacologiquement actives, les substance's antibactériennes et antif ongiques semblent ne pas pouvoir apporter un progrès à la thérapeutique actuelle. Il n'en est pas de même pour les substances antinéoplasiques et antivirales. Dans ces domaines d'activités, des animaux marins tels que éponges, ascidies ou bryozoaires ont, jusqu'à présent, fourni la plupart des substances biologiquement actives.
La spongouridine, issue de l'une d'entre elles, entre d'ailleurs dans la composition d'un médicament utilisé dans le traitement de la leucémie : elle fût isolée dès 1955 par Bergmann aux États-Unis [2] à partir de l'éponge Cryptotethia crypta et se trouve à l'origine de l'ARA-A (1- $\beta$-D-arabinosyl adenine) et de l'ARA-C ( $1-\beta-D$-arabinosyl cytosine) utilisés respectivement dans les traitements du virus de l'herpès, de l'encéphalite et de la leucémie ( figure I). L'activité de l'ARA-C résulte de sa ressemblance avec la cytidine, nucléoside constituant de l'ARN, dont il ne diffère que par la nature du reste glucidique : cette substance est un puissant inhibiteur compétitif lors de la réduction de l'ARN messager. La mise à la disposition du corps médical de l'ARA-C et de l'ARA-A est l'aboutissement d'un long effort de recherche puisque plus de 20 ans se sont écoulés entre l'isolement de la spongouridine et l'utilisation clinique de ses dérivés.

Plus récemment, l'examen systématique de l'activité antinéoplasique au National Cancer Institute (NCI) d'extraits d'organismes marins a permis à plusieurs équipes américaines de découvrir des substances antivirales et surtout antitumorales particulièrement prometteuses. L'activité antivirale et cytotoxique d'extraits d'ascidies* de la famille des Didemnidae fut décelée en 1978

* Ascidies (embranchement des Cordès, sous embranchement des Urocordès ou Tuniciers) : petits animaux marins sessiles (fixés au substrat) vivant solitaires ou en colonies.

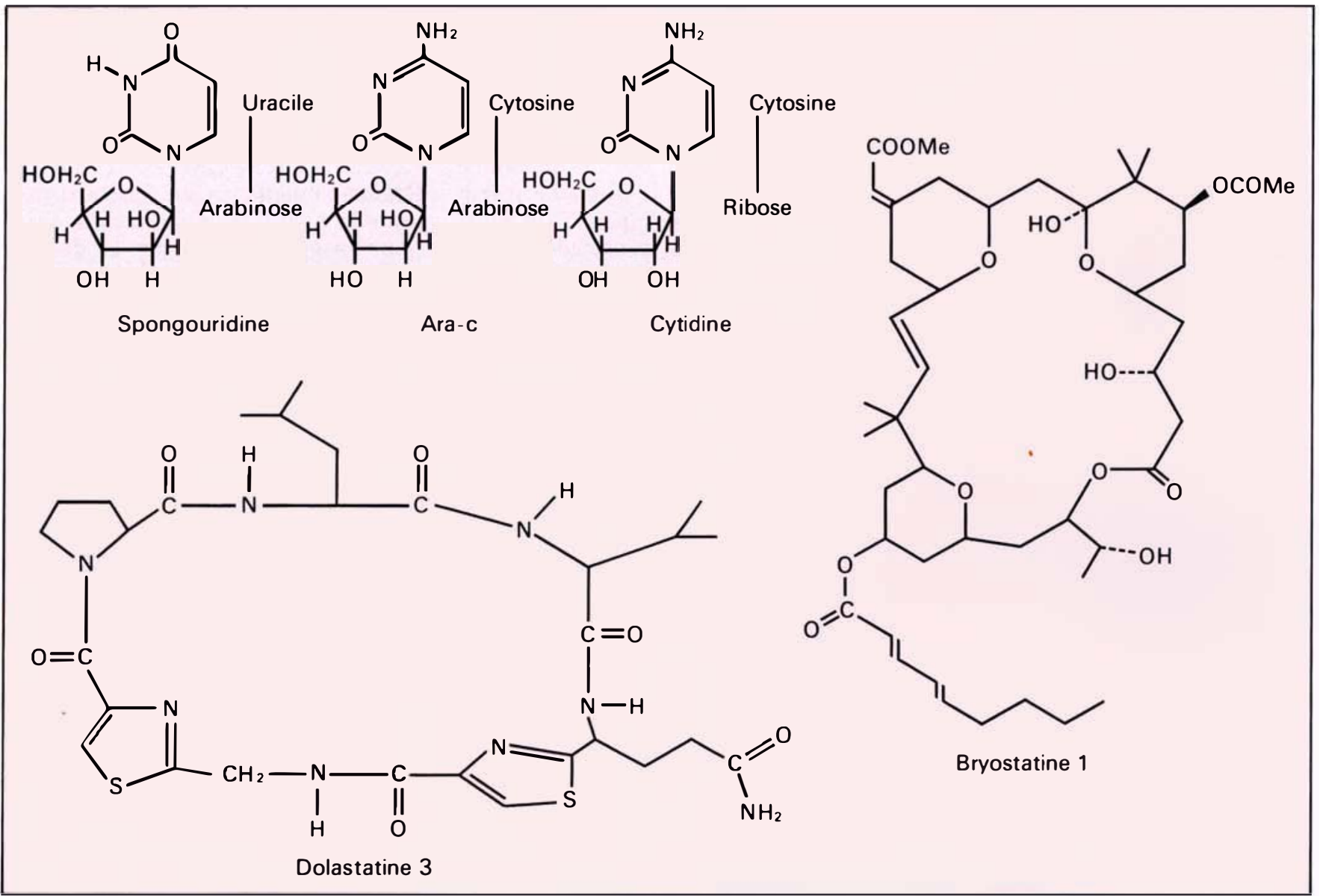

Figure I. Molécules marines à activités antivirales et antinéoplasiques. 
au cours d'une expédition de prospection en mer des Antilles [3]. $\mathrm{K}$ Rinehart et son équipe ont montré par la suite que l'activité antiherpétique (HSV-1 et $\mathrm{HSV}-2$ ) et l'activité antinéoplasique sur cellules leucémiques $\mathrm{P}_{388}$ sont associées à la présence dans ces extraits animaux d'une série de peptides courts cycliques (figure 2): les didemnines [4]. Ces composés sont d'autant plus intéressants que leurs différences d'activité sont fonction de modifications structurales mineures : la didemnine $A$ est un efficace inhibiteur de croissance de virus à $\mathrm{ARN}$ et à $\mathrm{ADN}(50 \%$ d'inhibition à $15 \mu \mathrm{g} / \mathrm{ml}$ sur herpès simplex type 2 et sur virus de Coxsackie) et la didemnine B un agent antinéoplasique puissant (tests in vivo sur cellules leucémiques $\mathrm{P}_{3} 88$ : T/C > 199 à $1 \mathrm{mg} / \mathrm{kg}$ ). A partir d'une autre ascidie coloniale, Eudistoma olivaceum, la même équipe [5] a isolé en 1984 une série de pseudopeptides bromés: les eudistomines. L'eudistomine C, par exemple, formée par condensation d'un tryptophane et d'une cystéine, inhibe le développement du virus de l'herpès $(\mathrm{HSV}-1)$ à des concentrations de l'ordre du nanogramme.

Un autre exemple de l'intérêt porté aux substances naturelles issues d'organismes marins pour la chimiothérapie du cancer nous est donné par l'équipe de G Pettit à l'Institut de recherche contre le cancer, dans l'Arizona (États-Unis). $100 \mathrm{~kg}$ d'un petit mollusque marin Dolabella auricularia furent nécessaires à cette équipe pour isoler $10 \mathrm{mg}$ d'une nouvelle série de 9 peptides cycliques: les dolasta-

Figure 2. Polysyncraton lacazei (cliché M. Whal), ascidie coloniale de la famille des Didemnidae. C'est de cette famille de Tuniciers que sont issues les molécules représentées sous le cliché, qui constituent des substances nouvelles dans la lutte contre les virus et contre le cancer.
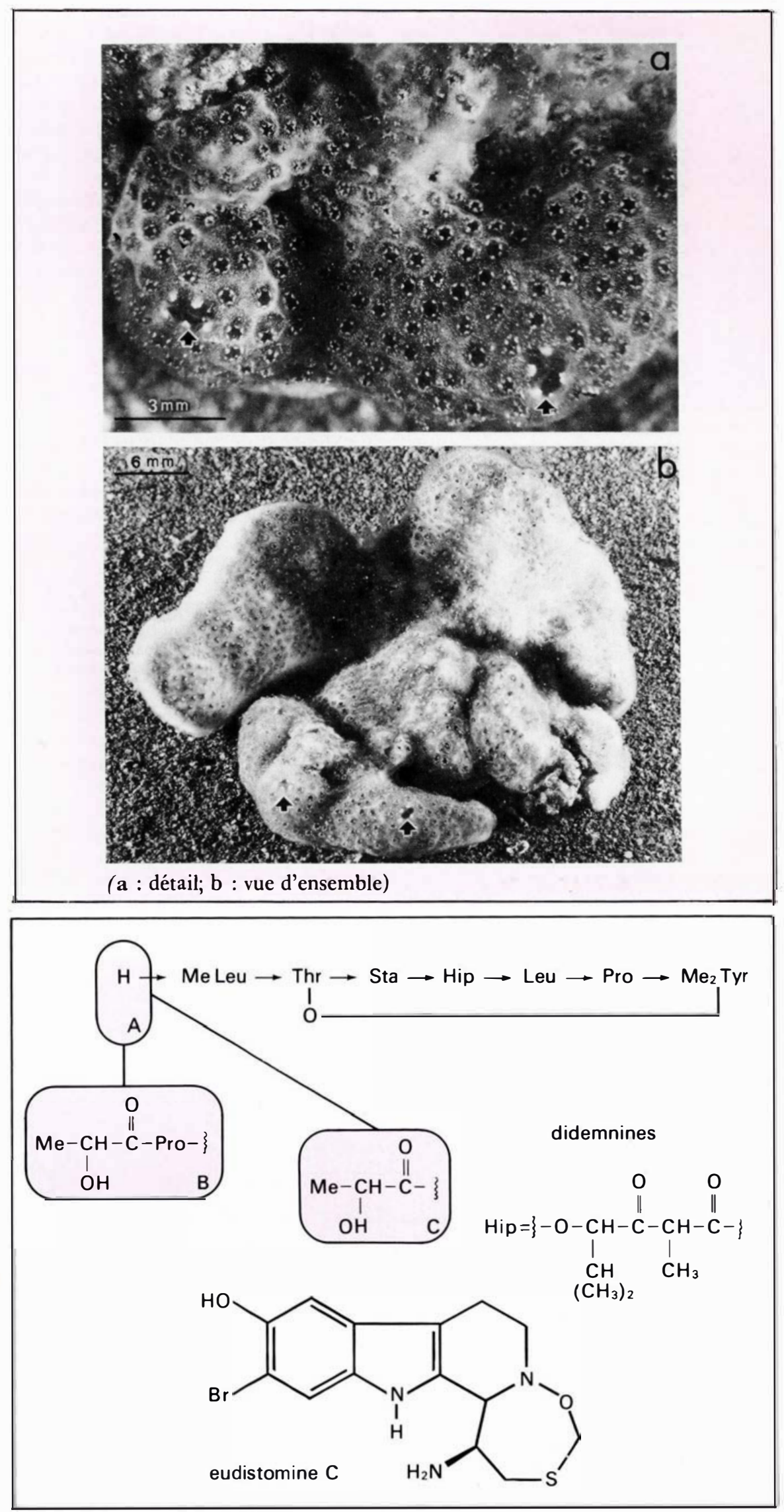
tines [6]*. La dolastatine 1 donne une réponse curative de $33 \%$ à une dose de $11 \mu \mathrm{g} / \mathrm{kg}$ ( $\mathrm{T} / \mathrm{C}_{240}$ ) sur mélanome $\mathrm{B} 16$ de souris, ce qui en fait un des agents antinéoplasiques les plus actifs connus jusqu'à présent ( figure 1).

Le cas des bryostatines étudiées par cette même équipe de l'Arizona est également exemplaire. Les études débutèrent en 1968 avec la première récolte du bryozoaire Bugula neritina, dans le golfe du Mexique. Les tests in vivo effectués avec les extraits de ces animaux incrustants ont révélé, très tôt, des taux d'activité relativement importants $(63 \%$ d'extension de vie à $30 \mu \mathrm{g} / \mathrm{kg}$ sur la leucémie $\mathrm{P}$ 388).

Ces résultats encourageants ont conduit G. Pettit et son équipe à

* Seule la structure de la dolastatine 3 a été publiee pour être d'ailleurs rapidement remise en cause par une équipe japonaise qui, synthétisant le composé décrit par Pettit, n'a pas retrouvé l'activité antitumorale correspondante. effectuer 2 nouvelles récoltes en $1978(118 \mathrm{~kg})$ et $1981(100 \mathrm{~kg})$ af in d'isoler en $\mathrm{I} 983$ les principes actifs : les bryostatines [7]. La bryostatine 1 , actuellement en essai préclinique est un agent antinéoplasique conduisant à une extension de vie de $68 \%$ à $40 \mu \mathrm{g} / \mathrm{kg}$ sur le sarcome $M_{531}$ de l'ovaire de souris ( figure 1).

\section{Agents cardiotoniques et cardiovasculaires}

Si de gros efforts sont consentis au niveau des tests et screenings d'antimicrobiens et d'antinéoplasiques, il n'en est pas de même pour les tests d'activités hypo ou hypertensives. De ce fait, assez peu de composés présentant de telles activités ont été isolés du milieu marin (figure 3). On peut néanmoins noter la présence, chez de nombreuses algues brunes, de la laminine et, chez les animaux marins, de dérivés de la $\beta$-phényléthylamine dans des ex-

\section{RÉFÉRENCES}

2. Bergmann W, Burke DC. The nucleosides of sponges III. Spongothymidine and spongouridinc. 7 Org Chem 1955; 20 : I50I-7.

3. Rinehart KL, Shaw PD, Shield LS, et al. Marine natural products as sources of antiviral, antimicrobial and antineoplastic agents. Pure and appl chem $198 \mathrm{I} ; 53: 595-617$

4. Rinehart KL, Gloer JB, Hugues RG, et al. Didemnins : antiviral and antitumor depsipeptides from a caribbean tunicate. Science $1981 ; 212$ : 933-5.

5. Rinehart KL, Kobayashi J, Harbour GC, Hugues RG, Mizsak SA, Scahill TA. Eudistomins $C, E, K$ and $L$, potent antiviral compounds containing a novel oxathiazepine ring from the caribbean tunicate Eudistoma olivaceum. $\mathrm{I} \mathrm{Am}$ Chem Soc 1984; 106 : 1524-6.

6. Pettit GR, Kamano Y, Brown P, Gust D, Inoue $M$, Herald $C L$. Structure of the cyclic peptide dolastatin 3 from Dolabella auricularia. 7 Am Chem Soc 1982; 104 : 905-7.

7. Pettit GR, Herald CL, Kamano Y. Structure of the Bugula nerita (Marine Bryozoa) antineoplastic component Bryostatin 3. J Org Chem 1983; $48: 5354-6$.

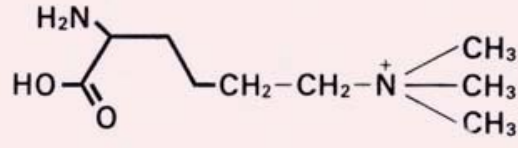

laminine

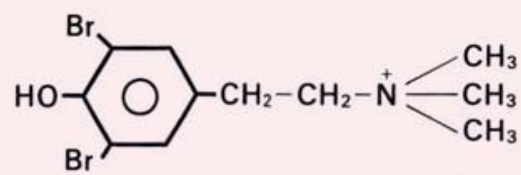

3,5-dibromo, 4-hydroxyphenethylmethylamonium

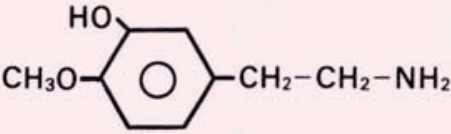

3-hydroxy-4 methoxyphenethylamine

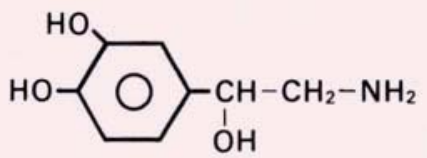

noradrénaline

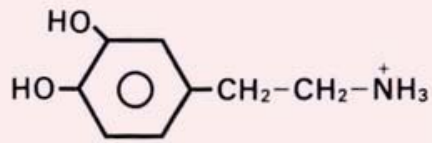

dopamine

Figure 3. Agents cardiovasculaires d'origine marine. 


\section{Les défenses chimiques des êtres vivants marins}
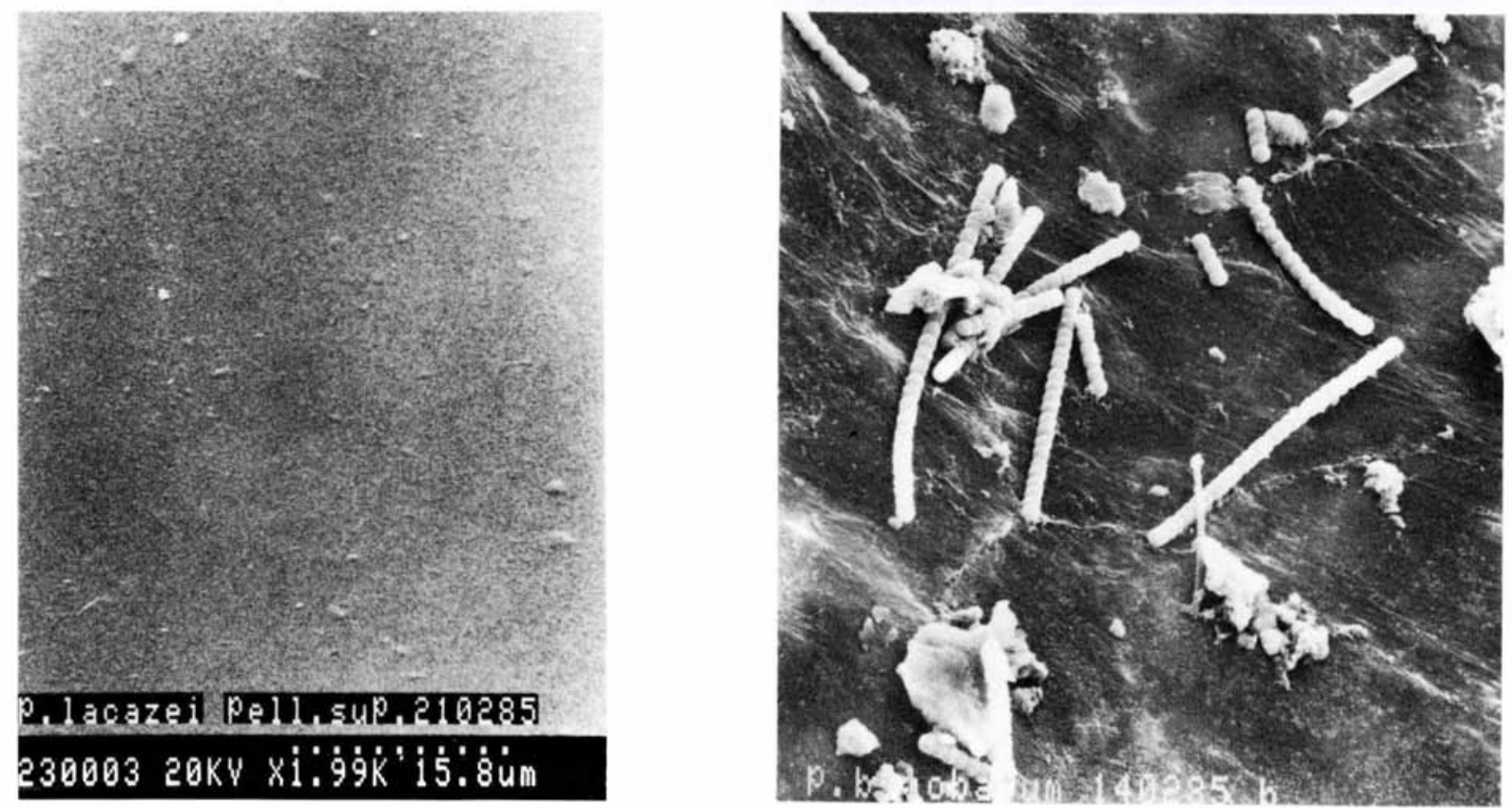

Des études écologiques faites sur les plantes terrestres ont montré que de nombreuses familles végétales, potentiellement comestibles, échappent totalement à la prédation des herbivores ou présentent un nombre restreint de prédateurs, spécifiques d'une espèce donnée. Le monde marin est également le siège de luttes incessantes entre proies et prédateurs. La fonction vectrice de l'eau où s'exercent difJusion et dispersion avec une grande facilité, favorise l'établissement de barrières chimiques dressées à l'aide de substances organiques qui sont désagréables au goût (antipalatabilité), toxiques ou qui réduisent la valeur nutritive des tissus. Les substances impliquées dans de tels mécanismes de défense (chimique) sont des métabolites dits secondaires (secondaires par opposition aux métabolites primaires - protéines, acide nucléique... - essentiels à la vie). Jadis considérés comme "quasi-gratuits", sous-produits ou déchets métaboliques à signification taxinomique, les métabolites secondaires ont été récemment reconsidérés sous l'angle écologique, biochimique et pharmacologique. Tanins, alcaloides et terpènes, les trois principales classes de métabolites secondaires présentent en commun des propriétés gustatives dissuasives et, bien que de structure moléculaire différente, offrent la particularité de posséder, sur un squelette carboné liposoluble, des groupements avides d'électrons qui vont soit induire des transformations chimiques, soit permettre à la molécule de se fixer sur un récepteur enzymatique. Mais la production des substances toxiques implique la mise au point, par les espèces vivantes concernées, d'adaptations biogénétiques qui suppriment le caractère néfaste des groupes électrophiles de ces molécules : si cette détoxification n'avait pas lieu, certaines espèces ne pourraient survivre ni à la synthèse ni au stockage de leurs propres armes chimiques.
Figure 5. I.es défenses chimiques des êtres vivants marins. Illustrations chez les Didemnidae (clichés

M Whal; microscopic électronique de la tunique : agrandissement $\times 1$ ooo pour Polysyncraton bilobatum, $\times 2000$ pour Polysyncraton lacazei). I.es photographies montrent la présence de spores et de bactéries sur l'espèce Polysyncraton bilobatum alors que Polysyncraton lacazei en est totalement dépourvue. Une telle constatation biologique permet de sélectionner l'espèce Polysyncraton lacazei pour la recherche de molécules impliquées dans les défenses chimiques (apparemment très efficaces) à potentialités thérapeutiques (antimitotiques, antibactériens, inhibiteurs enzymatiques...). 
traits hydrométhanoliques de l'éponge Verongia fistularis $(3,5$ dibromo-4 hydroxy-phenethylméthylamonium) [8] et du corail mou Nepthea sp (3 hydroxy-4 méthoxy-phenethylamine) [9].

L'analogie structurale de ces 3 composés avec des médiateurs chimiques naturels tels que la dopamine, la noradrénaline et l'adrénaline ou avec des substances de synthèse telle que l'isoprénaline peut expliquer leur activité hypotensive. Ces molécules possèdent en effet une affinité pour les récepteurs $\beta$-sympathiques de la noradrénaline et sont donc emmagasinées dans les sites tissulaires normalement réservés à cette hormone : libérés lors de la stimulation normale du système sympathique, il semble que ces "faux médiateurs" donnent lieu à une diminution des à-coups tensionnels et cardiaques.
Les prostaglandines ont plusieurs usages thérapeutiques, mentionnons : contraceptifs, prévention ou traitement des ulcères gastriques.

\section{Anti-inflammatoires et antidépresseurs}

Elles interviennent aussi dans les phénomènes de la régulation de la pression sanguine. Les propriétés anti-hypertensives d'un extrait aqueux d'une algue rouge Gracilaria lichenoïdes ont aussi permis à Gregson [10] d'isoler la première prostaglandine $\left(\mathrm{PGF}_{2 \alpha}\right)$ du monde végétal. Ces prostaglandines sont également impliquées dans les mécanismes de la douleur et de l'inflammation. Des recherches sont actuellement en cours pour trouver des molécules bloquant la formation des prostaglandines par action sur la phospholipase $\mathrm{A}_{2}\left(\mathrm{PLA}_{2}\right)$. La ma-

\section{RÉFÉRENCES}

8. Kaul PN. Compounds from the sea with actions on the cardiovascular and control nervous systems. Pharmacology of marine natural products. Fed proc I 981; 40 : 10-4.

9. Gregson RP, Lohr RR, Marwood JF, Quinn RJ. 3-hydroxy-4 methoxy phenethylamine, the cardioactive constituent of a soft coral. Experientia $1981 ; 37: 493-4$.

10. Gregson RP, Marwood JF, Quinn RJ. The occurence of prostaglandines $\mathrm{PGE}_{2}$ and $\mathrm{PGF}_{2 \alpha}$ in a plant: the red alga Gracilaria lichenoides. Tetrahedron Lett 1979; 4505-6.

11. Jacobs RS, Culver P, Langdon R, O'Brien T, White S. Some pharmacological observations on marine natural products. Tetrahedron $1985 ; 41$ (6) : 981-4.

12. Norton RS, Wells $R J$ J. A series of polybrominated bi-indoles from the marine blue-green alga Rivularia firma. I Am Chem Soc 1982; 104 : 3628-35.

13. Kazlauskas R, Murphy PT, Quinn RJ, Wells RJ. Aplysinopsin, a new tryptoplan derivative from a sponge. Tetrahedron Lett $1977 ; 6 \mathrm{I}-4$.

14. Higgs MD, Vanderah DJ, Faulkner DJ. Polyhalogenated monoterpenes from Plocanium cartilagineum from the Bristish coast. Tetrahedron

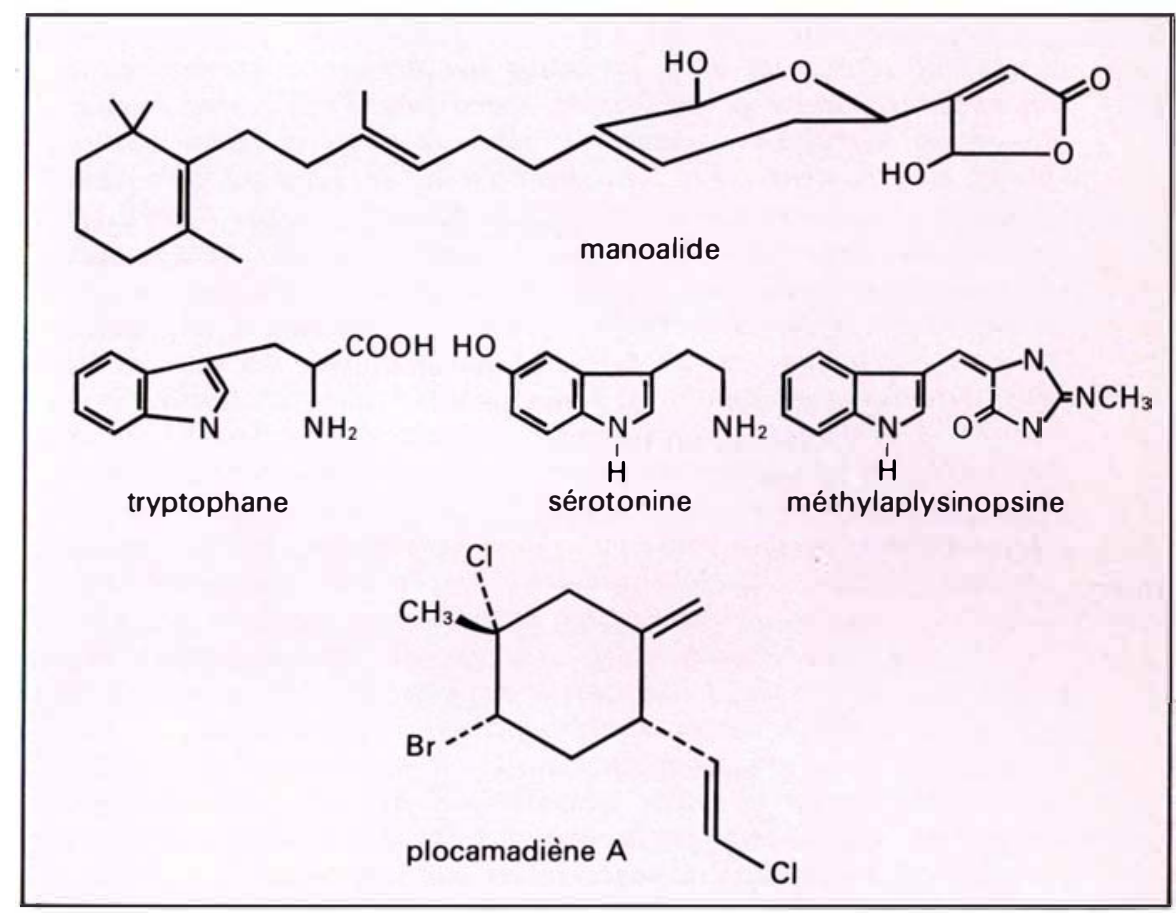

Figure 4. Substances à potentialités anti-inflammatoires et analgésiques isolées du milieu marin. 
noalide ( figure 4), sesterterpène original isolé de l'éponge Laffariella variabilis [11] (analgésique actif à $50 \mathrm{mg} / \mathrm{kg}$ ) est un agent anti-inflammatoire intéressant car, différent de la morphine et de ses dérivés, il représente une nouvelle classe de molécules utiles lors de l'étude de processus physiologiques dans lesquels la phospholipase $A_{2}$ intervient. Il en est de même pour un bi-indole polybromé, isolé de l'algue bleue Rivularia firma [12]. Deux autres dérivés indoliques, la méthylaplysinopsine et l'aplysinopsine ont été découverts dans une éponge Aplysinopsis reticulata [13]. La méthylaplysinopsine, dérivé du tryptophane, est un antidépresseur qui pourrait avoir les mêmes sites d'action que la sérotonine ( $f i$ gure 4). Cette hormone naturelle présente, en effet, une action importante sur le métabolisme du cerveau : en excès, elle provoque une stimulation de l'activité cérébrale alors qu'une déficience produit un effet dépresseur. De nombreux mono et diterpènes d'organismes marins ont également des activités d'antidépresseurs mais non spécifiques. Seule la plocamadiène A [14] extraite de l'algue rouge Plocamium cartilagineum, par son activité stimulante à longue durée, aurait un intérêt pour l'étude des récepteurs du système nerveux central.

\section{Médiateurs chimiques en milieu marin}

Face aux nombreuses espèces marines (500 000 environ), la recherche d'éventuelles substances naturellles à propriétés pharmacologiques constitue une tâche énorme et un tel criblage nécessite des moyens financiers et humains considérables. On peut y remédier par l'observation, in situ, des intéractions biologiques entre organismes marins (figure 5 , voir p. 367). Les écologistes nous ont appris que les organismes vivants utilisent pour se développer et se reproduire des médiateurs chimiques dont certains constituent des substances de défense que les producteurs utilisent pour se débarrasser de leurs concurrents. Ces substances de défense sont généralement des métabolites secondaires excrétés dans le milieu marin et ayant une action répulsive, toxique ou antimitotique. Par leurs actions sélectives sur les récepteurs biologiques, ces métabolites secondaires marins présentent non seulement un intérêt considérable en biologie et en biochimie, mais encore sont susceptibles d'importantes applications pharmacologiques. Ces médiateurs chimiques possèdent en effet les caractéristiques de bon nombre de médicaments, à savoir : activités spécifiques sur cibles biologiques et balance hydro-lipophile favorable.

I a plupart des sécrétions déf ensives des organismes marins contiennent des substances présentant, de par leurs structures, des caractères lipophiles et hydrophiles. Cela leur permet de diffuser à travers le milieu aqueux et de se disperser grâce à leurs groupements fonctionnels électrophiles, mais aussi de franchir les barrières cellulaires (membranes) par des parties de leur squelette plus lipophiles. I.es groupements fonctionnels électrophiles vont ensuite soit induire des dégradations chimiques, soit fixer la molécule sur un récepteur biochimique (activation, inhibition...).

\section{Conclusion}

La découverte d'une molécule nouvelle appartenant à une classe structurale connue, peut permettre d'orienter les recherches vers un type d'activité : c'est le cas des prostaglandines ou des dérivés du tryptophane. Mais ce type de travaux est surtout à même de fournir de nouveaux archétypes de molécules pharmacologiquement actives comme le démontre la découverte des didemnines ou des eudistomines. L'étude pharmacologique des intermédiaires de synthèse et des homologues (puisqu'il s'agit souvent de séries de composés) peut permettre ensuite de mieux comprendre les mécanismes d'action. Les études de pharmacologie marine, après une période d'essoufflement couvrant les années 70 , semblent prendre un nouvel essor au cours des années 80 .

Nous pensons que le début des années 90 verra l'apparition de nouveaux antitumoraux ou antiviraux d'origine marine en pratique médicale courante.

\section{Summary}

A survey of pharmaceutical potentialities in marine field based upon recent results is presented. Compounds like didemnins, eudistomins or dolastatins are described as models of novel structures with antineoplastic and antiviral activities. Marine constituents with cardioactive or antidepressive effects and usual drugs are compared.

The emergence of interdisciplinary concepts involving chemical defenses and biochemical mediators is also reported. These concepts provide a useful rationale for the discovery of novel physiologically-active metabolites with pharmacological interest. They open a new trend in marine research. 